\title{
Philosophische Schulen und ihre Kommunikationsräume im spätrepublikanischen und kaiserzeitlichen Rom
}

Wie bereits in Athen seit dem Beginn des ersten Jahrhunderts v. Chr., nachdem Sulla die Stadt verwüstet und dabei auch die Gebäude der Akademie vor dem Dipylon stark beschädigt hatte, ${ }^{\mathrm{I}}$ war auch im kaiserzeitlichen Rom mit dem Begriff der philosophischen ,Schule‘ nicht mehr notwendigerweise ein bestimmter, genau lokalisierbarer Ort verbunden. Die hellenistischen Philosophenschulen existierten unabhängig von ihren Athener ,Mutterhäusern' weiter, die - mit Ausnahme des epikureischen Gartens - verlassen oder verfallen waren. Sie hatten höchstens noch symbolische Bedeutung, als Erinnerungsorte für Gaststudenten: So lokalisiert Cicero die Diskussion des fünften Buchs von De finibus auf dem Gelände der platonischen Akademie, die er im Jahr 79 mit dem Bruder, einem Vetter und den beiden Freunden M. Piso und Atticus besuchte, nachdem man im Ptolemaion einen Vortrag des Antiochos von Askalon gehört hatte (fin. 5, If.). ${ }^{2}$ Cicero lässt Piso die Anlagen der Akademie als regelrechten lieu de mémoire beschreiben: Der Garten evoziert in ihm das Bild Platons, und ihn berührt die Vorstellung, dass Platons Nachfolger hier lehrten und dass Polemon auf dem offenbar noch sichtbaren Sessel gesessen habe. ${ }^{3}$

Die Szenerie, die Cicero zur Zeit der Abfassung des Dialogs - 35 Jahre später, im Jahr 45 v. Chr. - allenfalls verklärt, dürfte realistisch sein. In jedem Fall entsprach es gängiger Praxis, dass junge Angehörige der römischen Elite in den Bildungszentren des römischen Reichs Vorträge und Vorlesungen von Vertretern der wichtigen Philosophenschulen besuchten. Cicero hörte in Athen neben dem Akademiker Antiochos auch die epikureischen Schulhäupter Phaidros und Zenon von Sidon, in Rhodos den Stoiker Poseidonios, in Rom auch schon vor dem Athener Studienaufenthalt den Akademiker und Scholarchen Philon von Larissa und bereits damals den Epikureer Phaidros. 4 Diese professionellen Philosophen unterrichteten offenbar - wie die Rhetoren - in öffentlichen oder privaten, wohl gemieteten Räumen..$^{5}$ Man brauchte keinen bestimmten Hain, keine Säulenhalle, keinen Garten, um ein Lehrsystem weiter tradieren und ausbilden zu können. ${ }^{6}$ Dies änderte sich auch nicht, als Mark Aurel im Jahr I76 in Athen vier bezahlte Lehrstühle für die vier Haupt-

I Dazu Görler (I994) 777.

2 Zum Ptolemaion als zeitweiligem Sitz der Akademie vgl. Görler (I994) 778.

3 Die Szene analysiert Haake (2007) I3-I6, der zum Vergleich Herakleides Kritikos I,I heranzieht.

4 Dazu Gawlick u. Görler (I994) Ioogf.

5 Dazu Hadot (2003) 49f.

6 Dies wird auch aus der Wahl der Szenerie der platonischen Dialoge deutlich, die Platon an den unterschiedlichsten Orten stattfinden lässt; die Akademie war zu Sokrates' Lebzeiten ja noch nicht gegründet. 
schulen einrichten ließ.7 Die Begriffe der Akademie, des Peripatos oder Lykeion, der Stoa (lat. porticus) und des Kepos (lat. öfter hortulus) bezeichneten zum Zeitpunkt ihres Imports in Rom kaum mehr als die ursprünglich in diesen Gebäuden entwickelte Lehre, zu der

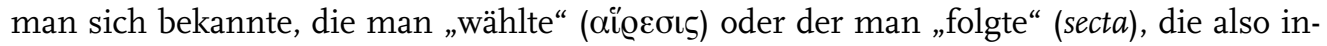
haltlich definiert war.

Die Namen der Philosophenschulen standen also spätestens im spätrepublikanischen und kaiserzeitlichen Rom nicht mehr für geographisch und topographisch festgelegte Orte, sondern für nicht-materielle Denkräume, in denen Wissen und Erkenntnisse verhandelt und Erfahrungen ausgetauscht, mithin: kommuniziert wurden. ${ }^{8}$ Solche kommunikativen Handlungen finden nicht notwendigerweise in einem Raum statt, der durch eine bauliche Struktur räumlich beschränkt sein muss, vielmehr können die Teilnehmer/innen eines kommunikativen Prozesses auch durch lokale Distanz getrennt sein. Auch der schriftliche Austausch von Informationen innerhalb eines Kreises von Schreiber/innen und Adressat/innen generiert einen Kommunikationsraum. So konstruiert beispielsweise Senecas Briefwechsel mit Lucilius, in dem eine Abfolge von Fragen und Antworten vorausgesetzt - auch fingiert - und Informationen und Ratschläge ausgetauscht werden, einen literarischen Denk- und Kommunikationsraum.

Neben der Möglichkeit, durch den Austausch von philosophischer Literatur auch virtuelle Räume der Philosophie herzustellen, spielten aber für die weitere Entwicklung und Verbreitung der hellenistischen Philosophenschulen in Rom dennoch auch konkrete Räume eine gewisse Rolle. Dies ist zumindest der Eindruck, der in den Texten Ciceros und Tacitus' für die späte Republik und frühe Kaiserzeit vermittelt wird: Philosophische Diskussionen sind fast durchweg in einem privaten Raum, in einem Haus, Garten oder Zimmer lokalisiert, die damit in gewissem Sinn die Funktion der Institutionen einer philosophischen Schule übernehmen. Die Lokalität, in der ein Dialog stattfindet, wird in der Regel genannt oder sogar genauer beschrieben, so dass der Eindruck entsteht und oft wohl auch entstehen soll, dass der Szenerie eine bestimmte Bedeutung zukommt. 9 In meinen folgenden Ausführungen möchte ich daher die Frage stellen, welche Funktionen die Räume, in denen philosophische Kommunikation stattfindet bzw. in denen sie lokalisiert wird, zu erfüllen haben, welche impliziten Botschaften, welche Semantik, welche Ideologien mit ihnen verbunden werden können oder sollen.

Raum für die Kommunikation philosophischer Inhalte boten im spätrepublikanischen und frühkaiserzeitlichen Rom nicht nur die Lokalitäten, in denen Philosophie professionell -

7 Cass. Dio 7I,32,I. Dazu Hadot (2003) 52.

8 Unter Kommunikation wird hier im ganz allgemeinen Sinn eine sprach- und/oder textbasierte Interaktion verstanden, die der Vermittlung von Informationen (Wissen, Erkenntnis, Erfahrung) dienen soll; die Frage, ob ein solches kommunikatives Handeln erfolgreich ist, spielt für die vorliegende Fragestellung keine Rolle. Für die Möglichkeit einer solchen offenen Definition vgl. Meggle ( ${ }^{2}$ I997) 7 f. Die Kommunikationssituation kann auch eine Lehr- bzw. Unterrichtssituation sein.

9 Zur Bedeutung der Szenerie in den ciceronischen Dialogen vgl. Zoll (I962) 96-IO5, der die Frage am Beispiel von Ciceros De oratore diskutiert. 
gegen Honorar - gelehrt wurde, sondern auch die Privathäuser der römischen Elite, die gerne sogenannte Hausphilosophen beschäftigte, eine Praxis, die bereits die hellenistischen Herrscher pflegten. ${ }^{\text {IO }}$ Diese wurden mit der Erziehung der Söhne des Hauses betraut und übernahmen bisweilen die Funktion von Vertrauten und Betreuern der Familie. Nicht zuletzt sollten sie auch das Bemühen der Hausherrn um philosophische Bildung und griechische Kultur dokumentieren. ${ }^{\text {II }}$ Der jüngere Cato wird in der Literatur oft in der Gesellschaft von griechischen Philosophen dargestellt, und nach Plutarch standen ihm bei seinem Freitod der Stoiker Apollonides und der Peripatetiker Demetrios zur Seite. ${ }^{12}$ Cicero beherbergte in seinem Haus in Rom den Stoiker Diodotus, Caesars Schwiegervater L. Calpurnius Piso Caesoninus den Epikureer Philodem. Oktavian bzw. Augustus hatte die Stoiker Athenodor von Tarsos und Areios Didymus als private Lehrer und auch als politische Berater in seinem Haus. ${ }^{13}$ So konnte also das Wohnhaus eines römischen Senators und auch des Kaisers zum Kommunikationsraum philosophischer Lehren werden bzw. solche Räume enthalten. ${ }^{4}$

Auffällig ist, dass Cicero seine Dialoge nie in städtischen Häusern stattfinden lässt, sondern nur in Villen am Stadtrand ${ }^{15}$ oder außerhalb Roms, in De finibus 5 in Athen. Auch lässt er keine Hausphilosophen auftreten: ,Sein' Stoiker Diodot wird nie als philosophischer Experte inszeniert, geschweige denn, dass Cicero dem Epikureer Philodem eine Stimme geben würde. ${ }^{6}$ Vielmehr tauschen in den von ihm konstruierten Kommunikationsräumen die Angehörigen der römischen Elite philosophisches Wissen aus, und bekanntlich tun sie dies nur dann, wenn sie von ihren politischen Pflichten entbunden sind. Philosophische Gespräche sollten offensichtlich als Freizeittätigkeit der senatorischen Nobilität gelten, die unabhängig von der Situation des professionellen und hauseigenen Lehrbetriebs ausgeübt wurde. Dabei hatten jedoch politische Fragen durchaus auch ihren Platz: Im geschützten Raum der Landhäuser, in Bibliotheken oder Wandelhallen lässt Cicero seine Figuren immer wieder auch die politische Situation der Gegenwart kommentieren, womit er die in den Proömien im eigenen Namen geäußerten Klagen von dritter Seite bestätigen lässt. Nach Hermann Strasburger können die Spätdialoge geradezu als „Aufruf gegen die Herrschaft Caesars“ gelesen werden. ${ }^{\mathrm{I7}}$ Die Vermittlung einer im Kon-

Io Dazu Rawson (1989); Gehrke (I998).

II Eine Liste der ,Hausphilosophen und ihrer Patrone stellt Malitz (I988) I55f. zusammen; vgl. auch Griffin (1989) 3-5.

I2 Plut. Cato min. 68,5 (vgl. 65,II; 66,6-8; 69,I; 70,I). Zu seiner Entourage gehörten auch die Stoiker Antipater von Tyros und Athenodoros Cordylion (Plut. Cato min. 4,If.; Io,I-3; I6,I); vgl. auch 6,3; 20,2. Dazu Zadorojnyi (2007), bes. 2 I7 mit Anm. 5.

I3 Dazu Maier (I985) 42-47; Rawson (I989) 243-246.

I4 Die entsprechend genutzten Räume wurden offenbar gerne auch mit Büsten und Mosaiken mit Darstellungen von Philosophen als solche markiert. Vgl. dazu Lorenz (1965).

I5 So in rep. I,I4: in hortis. Den Hinweis verdanke ich Peter L. Schmidt.

I6 Die Tatsache, dass sein persönlicher Feind Piso sein Haus in Herculaneum Philodem als Wirkungsstätte und Kommunikationsraum zur Verfügung stellte, nutzte Cicero vielmehr als Anlass zu bissigstem Spott (Pis. 68-72). I7 Strasburger (1990); vgl. auch Wassmann (I996) I-I8 mit einer Diskussion der Forschungstradition, in der Philosophie und Politik auch als Alternativen gesehen werden (Manfred Fuhrmann, Klaus Bringmann u.a.). 
text philosophischer Theorien entwickelten politischen Botschaft konnte offenbar besser funktionieren, wenn ihre Absender nobiles und nicht profesionelle Philosophen waren, und sie war unverfänglicher, wenn die Szenerie örtlich und zeitlich entrückt war, im Fall von De re publica in die Zeit des sogenannten - von Cicero inszenierten - ,Scipionenkreises' ${ }^{18}$

Die von Hermann Strasburger prominent vertretene Idee einer subversiven Inanspruchnahme der Philosophie lässt sich dadurch stützen, dass man in Rom seit dem zweiten vorchristlichen Jahrhundert geneigt war, einer philosophischen Diskussion tagespolitische Aktualität und dabei eine regimekritische bzw. - je nach Perspektive - eine staatsgefährdende Intention zuzuschreiben. Beginnend im Jahr I6I v. Chr., als zum ersten Mal griechische Philosophen und Rhetoren aus Rom vertrieben wurden, setzte sich die Tradition, professionelle Philosophen aus der Stadt auszuweisen, bis in die frühe Kaiserzeit fort. ${ }^{99}$ Auch nachdem die Lehren der hellenistischen Philosophenschulen längst zum Bildungsgut der politischen Elite gehörten, konnte das philosophische Wissen offenbar immer wieder als Gefahr für den regierenden Prinzeps wahrgenommen werden. Das erste nachchristliche Jahrhundert wurde zur Blütezeit der sogenannten Philosophen-Märtyrer ${ }^{2 \circ}$ Se- $^{-}$ neca, Rubellius Plautus, Thrasea Paetus, Helvidius Priscus, Barea Soranus, Musonius Rufus u.a., die unter Nero, Vespasian und Domitian des politischen Widerstands gegen den Kaiser angeklagt und verurteilt, exiliert oder zur Selbsttötung gezwungen oder ermordet wurden. Sie sind keine professionellen Philosophen - auch nicht Seneca - in dem Sinn, dass sie philosophische Denksysteme entwickelten und hauptberuflich lehrten, sondern - wie die Gesprächspartner der ciceronischen Dialoge - römische nobiles, ${ }^{21}$ die sich als Vertreter der stoischen Position zu erkennen gaben. ${ }^{22}$ Seneca trat zudem als Verfasser philosophischer Schriften in Erscheinung; von Thrasea Paetus ist eine Lobschrift auf den jüngeren Cato bezeugt, in der er dessen Leben und Tod heroisiert habe. ${ }^{23}$ Wichtig blieb aber offenbar immer auch die mündliche Kommunikation. Helvidius Priscus, Barea Soranus und eine Reihe weiterer Senatoren werden von Tacitus, Cassius Dio und Sueton zu Gesinnungsgenossen des Thrasea Paetus gemacht, die sich regelmäßig auch in ihren Privathäusern trafen. ${ }^{24}$ Vorstell-

I8 Zum politischen Hintergrund dieser ,Entrückung، vgl. Zetzel (I995) 5-8; ähnlich zu De legibus Eigler (I996), bes. I42.

I9 Die ,Philosophengesandtschaft' des Jahres I55 wurde nicht vertrieben, erweckte jedoch offenbar bestimmte Ängste. Dazu Haake (2007) IO6-II7.

20 Maier (I985) I70 spricht von „philosophisch-politische[n] Märtyrer[n]“.

2I Dazu gehören nun auch Angehörige der Reichsaristokratie und soziale Aufsteiger, wie der Paduaner Thrasea Paetus und sein Schwiegersohn Helvidius Priscus. Dazu Malitz (I988) I68 und I73f.

22 Wie Griffin (I989) 8-Io und Trapp (2007/22008) passim, bes. I35 ff., betonen, war die stoische Lehre in Rom am erfolgreichsten und wurde - nicht erst in der Kaiserzeit - zur Leitphilosophie. Vgl. auch Malitz (I988) I52f. und I6of., der jedoch zu Recht auch die Bedeutung der epikureischen Schule betont.

23 Sie war eine der Hauptquellen von Plutarchs Cato min.; dazu Tandoi (I966).

$24 \mathrm{Zu}$ Thraseas Vertrauten gehörte wohl auch der Satiriker Persius. Zu den Quellen vgl. Malitz (I988) I62 f.; Radice $u$. Ramelli (2008) I375-I394 und I406-I4I6. 
bar sind "römische Salons“ oder jours fixes, wo philosophische Positionen und auch politische Meinungen diskutiert wurden. ${ }^{25}$

Gerne spricht man auch von einer stoischen Opposition unter Nero, als deren Hauptexponenten der späte Seneca und Thrasea Paetus gelten können, ${ }^{26}$ sowie einer solchen unter den Flaviern, zu der Annaeus Cornutus, Musonius Rufus, Epiktet, Helvidius Priscus, Arulenus Rusticus und Herennius Senecio gerechnet werden. ${ }^{27}$ Damit ist die Vorstellung verbunden, dass die stoische Lehre die Grundlage und damit auch eine philosophische Legitimation bot, um zwar die monarchische Staatsform des Prinzipats zu akzeptieren, wie dies Seneca in De clementia ausführt, ${ }^{28}$ die aber gleichzeitig die moralische Kritikfähigkeit jedes Individuums und insbesondere - in der Tradition Platons - die moralische Integrität des Herrschers einforderte. ${ }^{29}$

Tacitus zeichnet in den Annalen tatsächlich ein recht homogenes Bild von den genannten stoisierenden Aristokraten und ihrem Verhalten gegenüber dem Kaiserhof, den Höflingen und korrumpierten Senatoren. Sie werden als rechtschaffene Männer gezeichnet, als viri boni, die versuchen, bestimmte ethische Werte zu bewahren, zu vertreten und zu leben..$^{\circ}$ In der informellen Anklage des Cossutianus Capito gegenüber Nero wird Thrasea mit dem jüngeren Cato verglichen, dessen Biographie Thrasea verfasst hatte..$^{3 \mathrm{I}}$ Der Vergleich klingt im Munde des Anklägers wie eine Warnung: Cato ist nicht nur der Vertreter rigider Prinzipien, sondern auch der Kämpfer par excellence für die republikanische Freiheit. Da Cato ein erklärter Stoiker war, impliziert der Vergleich auch eine Art Diadochie als (inoffizielles) Schulhaupt: Thrasea ist jetzt der Anführer des stoischen Widerstands gegen das Regime und damit der wichtigste Kämpfer für die senatorische Freiheit oder der härteste Gegner des Kaisers.

25 So Maier (1985) 59; vgl. auch unten Anm. 26.

26 Vgl. z.B. Maier (I985) I49ff.; Malitz (I988) I6Iff. - Maier (I985) 6o bezeichnet die circuli („Salons“ oder „jours fixes“) der stoischen Senatoren, namentlich des Thrasea Paetus und der Familie der Annaei, zu der neben Seneca und seinen Brüdern und dem Neffen Lucan auch Cornutus gehört, als „Brutstätten aristokratisch-subversiver Vorstellungen“. Dazu Raaflaub (I987) I4-I6, der sich gegen eine undifferenzierte Vorstellung einer ,stoischen Opposition` ausspricht.

27 Vgl. dazu den Überblick bei Malitz (1988) und Fuhrer (20I2).

28 Dazu Raaflaub (I987) 34; Braund (2009) 64-76.

29 Die grundsätzlich pro-monarchische, aber moralisierende Tendenz der stoischen Senatoren heben Malitz (I988) I6I und Raaflaub (I987) 27 hervor. Shaw (I985) sieht die stoische Philosophie sogar als Grundlage für die Ideologie monarchischer Herrschaftssysteme, die sich in hellenistischer Zeit etablierten; Roller (200I) 64ff. geht noch weiter und schreibt der kaiserzeitlichen Stoa, namentlich Senecas Schriften, die Intention zu, der aristokratischen Elite Roms eine neue ethische Grundlage vermitteln und damit auch politisch stabilisierend wirken zu wollen.

30 Dass Thrasea ein Stoiker war, wird nicht gesagt, dies wird aber aus Tacitus' Beschreibung seines Verhaltens und der Definition seiner ethischen Prinzipien deutlich; vgl. dazu Malitz (I988) I67f.

3I S. o. S. 244 mit Anm. 23; vgl. bereits Mart. I,8. Zur Anklage des Cossutianus Capito, der (gemäß Tacitus) in seiner Rede Thrasea als sein eigenes Gegenbild stilisiert und damit indirekt Nero charakterisiert, vgl. Galtier (2002) 3I2f. 
Im Gegensatz zu Cato kämpft Thrasea aber nicht bis zum Schluss an vorderster Front. Er beteiligt sich zwar, ähnlich wie Seneca, jahrelang an den politischen Prozessen, zieht sich dann aber mehr und mehr von seinen Aktivitäten im Senat und aus dem Umkreis des Kaiserhofs zurück, was ihm von seinem Ankläger auch zum Vorwurf gemacht wird - mit Erfolg: ${ }^{2}$ Nero bestellt den Senat ein, der in einer Gerichtsverhandlung im Tempel der Venus Genetrix auf dem Caesarforum ${ }^{33}$ über das Verhalten von Thrasea und Barea Soranus und auch anderer Gleichgesinnter urteilen soll. Da Thrasea die Situation im Senat so einschätzt, dass eine Verteidigungsrede keinen Erfolg haben würde,34 bleibt er zu Hause. Dies lässt Tacitus sein Lesepublikum aber erst nach der Erzählung von der dramatischen Gerichtsszene wissen, in der Barea Soranus und seine Tochter Servilia zum Tode verurteilt werden: Das Urteil gegen Thrasea wird ihm nämlich vom Quaestor der Konsuln in seinem Haus überbracht.

Tacitus lässt also auf die Szene im Raum des Tempels auf dem Caesarforum eine Episode in einem senatorischen Haus folgen. 35 Sie zeigt Thrasea im Kreis seiner Getreuen und der Familie sowie in der Anwesenheit des Kynikers Demetrius, mit dem er die Frage nach der Beschaffenheit der Seele und nach ihrer Verbindung mit dem Körper bzw. ihrer Trennung vom Körper diskutiert. Man hält sich dabei „im Garten“ auf, 36 und nach der Verkündigung des Urteils durch einen Getreuen, der offenbar in der Gerichtsverhandlung im Senat anwesend war, geht Thrasea in die Porticus, wo ihn der Quaestor erwartet. Dann zieht er sich zusammen mit seinem Schwiegersohn Helvidius Priscus und Demetrius in sein Schlafzimmer zurück, um sich die Pulsadern aufzuschneiden, was er als Trankopfer für Iuppiter liberator verstehen will, und langsam zu sterben. ${ }^{37}$

Die Szenerie evoziert die Vorstellung, dass Thrasea sein Haus in Rom zu einem Kommunikationsraum für philosophische Fragen gemacht hatte, in dem sich öfter „promi-

32 Tac. ann. I6,2If.

33 Ann. I6,27,I; dazu Koestermann (I968) 392. Zur kommunikativen Funktion dieses Tempels vgl. auch den Beitrag von Jan Stenger in diesem Band.

34 Ann. I6,26. Gemäß der Terminologie von Joachim Knape (in diesem Band, S. I33 f.) sind dies „Settingbedingungen“, die in diesem Fall klar gegen einen Auftritt in der Senatsversammlung sprachen.

35 34. (I) Tum ad Thraseam in hortis agentem quaestor consulis missus vesperascente iam die. inlustrium virorum feminarumque coetus frequentes egerat, maxime intentus Demetrio Cynicae institutionis doctori, cum quo, ut coniectare erat intentione vultus et auditis, si qua clarius proloquebantur, de natura animae et dissociatione spiritus corporisque inquirebat, donec advenit Domitius Caecilianus ex intimis amicis et ei quid senatus censuisset exposuit. ... 35. (I) Tum progressus in porticum illic a quaestore reperitur, laetitiae propior, quia Helvidium generum suum Italia tantum arceri cognoverat. accepto dehinc senatus consulto Helvidium et Demetrium in cubiculum inducit: porrectisque utriusque brachii venis, postquam cruorem effudit, humum super spargens, propius vocato quaestore, libamus inquit, Iovi liberatori. specta, iuvenis; et omen quidem dii prohibeant, ceterum in ea tempora natus es, quibus firmare animum expediat constantibus exemplis." (2) post lentitudine exitus graves cruciatus adferente, obversis in Demetrium **** (oculis Koestermann). Text: Heinrich Heubner (Bibliotheca Teubneriana).

36 Hier stellt sich die Frage, ob in hortis den Garten des senatorischen Stadthauses oder ein Haus am Stadtrand bezeichnet, wie dies in rep. I,I4 der Fall ist (s.o. Anm. I5).

37 Zur Szene (auch zur Anspielung auf Plat. Phd. II7a-II8) vgl. Gnilka (I979) Iof.; Billerbeck (I979) 45f.; Radice u. Ramelli (2008) I370 und I40I. 
nente Männer und Frauen“ (inlustres viri et feminae) aufhielten: Angehörige der sozialen Elite, auch ein ,hauptberuflicher' Philosoph, den Thrasea in der Sterbeszene neben sich haben will und dem in Tacitus' Darstellung sein letzter Blick (?) gilt. ${ }^{8}$ Im Gegensatz zu Cicero verlegt Tacitus den philosophischen Dialog - wie ja auch seinen Dialogus - in die Stadt. Auch die Personenkonstellation ist nicht ciceronisch: Ein Mitglied der römischen Elite diskutiert in seinem Wohnhaus im Kreis seiner Familie und Freunde, darunter auch Frauen, mit einem professionellen Philosophen über das alte Thema der Unsterblichkeit der Seele, das in seiner gegenwärtigen Situation auch eine lebensweltliche Relevanz aufweist. Gemäß Tacitus' Darstellung inszeniert Thrasea das philosophische Gespräch gleichzeitig mit und damit auch in Konkurrenz zu der Senatssitzung, in der die Anklage gegen ihn verhandelt wird, und macht dadurch deutlich, dass er den Kommunikationsraum in seinem Privathaus dem Versammlungsort der Magistrate vorzieht. Er stellt die Diskussion einer philosophischen Frage mit Demetrius, ausgerechnet einem Kyniker, in der Wertehierarchie über die Debatte des politischen Gremiums.39

Thrasea wird dabei sowohl als Sokrates stilisiert, der ebenfalls von einem politischen Gericht verurteilt und zur Selbsttötung gezwungen wurde, wie auch als zweiter Cato, der vor dem Freitod den platonischen Phaidon gelesen haben soll, wie auch als zweiter Seneca, der sich in Tacitus' Erzählung nach der Verurteilung die Pulsadern aufschneidet und ebenfalls langsam stirbt und dem Iuppiter liberator eine Libation darbringt. $4^{\circ}$ Durch diese Typisierung in der Sterbeszene wird Thrasea zu einem weiteren Philosophen-Märtyrer, zur Symbolfigur einer Gruppe von Senatoren, die sich in Tacitus' Darstellung dadurch von anderen abgrenzen, dass sie ihr Verhalten und auch ihr politisches Handeln an den ethischen Normen - dem sittlich Guten, dem honestum - ausrichten, die die Stoa für gültig erklärt.

Man braucht dabei nicht von politischer Opposition in dem Sinn zu sprechen, dass diese Senatoren Widerstand gegen das politische System des Prinzipats oder gegen die Person des Kaisers geleistet hätten. Die subversive Intention ist vielmehr eine Zuschreibung der Ankläger, wie sie Tacitus referiert. ${ }^{\mathrm{I}}$ Was Tacitus am Schluss der Annalen und auch an den anderen Stellen, wo er philosophierende Politiker oder politisierende Philosophen auf-

38 Oculis ist allerdings ergänzt (s.o. Anm. 35). Der überlieferte Text der Annales bricht hier - wohl ungewollt effektvoll ab; dazu Koestermann (ig68) $409 \mathrm{f}$.

39 Maier (1985) I90 bezeichnet Demetrius als "gelebte Alternative“ zur „Zivilisation des römischen Imperiums“; dagegen spricht allerdings Tac. hist. 4,40,3; dazu Billerbeck (I979) 46f. - Man könnte in dem „Garten“, den Thrasea verlässt, um in die porticus zu gehen, einen Verweis auf den epikureischen Kepos sehen, in dessen Abgeschiedenheit sich Thrasea zurückzieht, in der porticus einen Verweis auf die Stoa, zu der er sich im öffentlichen Leben bekennt. Das diskutierte Thema verweist auf Platons Phaidon. Anwesend ist ein Kyniker. So ergibt sich eine Zusammenstellung unterschiedlicher philosophischer Lehren, mithin ein Bekenntnis zur Philosophie in einem umfassenden Sinn, die im Kommunikationsraum von Thraseas Haus ihren Ort hat.

40 Ann. 15,64,4. Zu dieser Parallelität in Tacitus' Darstellung der beiden Biographien vgl. Vielberg (I987) 48-70, der für eine differenziert positive Wertung durch Tacitus plädiert. Zur Traditionskette der ultima verba vgl. Gnilka (I979) 8-II.

4I Vgl. z.B. ann. I4,57. Ähnlich Raaflaub (I987) 27. 
treten lässt und zu Märtyrern macht, zur Darstellung bringt - so auch in den Historiae -, sind nicht die Agitationen und das Scheitern einer organisierten politischen Widerstandsbewegung. Demonstriert wird vielmehr die Machtlosigkeit dieser Politiker, die sich mit ihren moralischen Handlungsnormen nicht bloß in einer allgemeinen Weise an den altrömischen mores orientierten, sondern an Begriffen und Werten, die im theoretischen System der Stoa festgeschrieben waren. Der alte Vorwurf, der immer wieder gegen die Philosophen - gerade gegen Seneca - erhoben wurde, nämlich dass sie den ethischen Anforderungen ihrer Lehre nicht entsprechen konnten, ${ }^{42}$ spielt hier keine Rolle, er wird vielmehr implizit zurückgewiesen. Tacitus lässt die Stoiker durchaus gemäß den von ihnen akzeptierten Grundsätzen leben und handeln. Genau dieses Verhalten führt jedoch zu ihrem Verderben, zum Exil oder Tod. Thrasea symbolisiert also das Scheitern des vir bonus et studiosus sapientiae unter einem Kaiser, der die virtus nicht als Handlungsorientierung, sondern als Odium definiert, das es auszurotten gilt.43

Tacitus macht in seiner Erzählung deutlich, dass unter Neros spätem Regime philosophische Fragen ernsthaft nur noch im begrenzten Raum einer senatorischen domus diskutiert werden konnten. Er greift dabei auf die von Cicero fast beschworene Vorstellung zurück, dass die Senatoren und Angehörigen der sozialen Elite Gespräche führten, in denen sie - wie beispielsweise in De finibus - die „Grenzen von Gut und Böse“ schulmäßig definierten. Tacitus kann zudem die von Seneca in den philosophischen Schriften beschriebenen Entwürfe eines guten Lebens als bekannt voraussetzen, die dieser an die Adresse von Bekannten und Verwandten, die als Senatoren und kaiserliche Beamte tätig waren, gerichtet hatte. Mit der Figur Thraseas inszeniert Tacitus also den Erfolg der von Seneca literarisch kommunizierten stoischen decreta et praecepta, die Umsetzung der Anleitung zur Selbstvervollkommnung mit dem Ziel der inneren Unabhängigkeit von äußeren Gegebenheiten, letztlich auch von den konkreten Lebensumständen und der geschichtlichen Wirklichkeit.44 Tacitus zeigt also zwar das Scheitern der Philosophen-Politiker auf, er stellt damit aber nicht die Gültigkeit der philosophisch begründeten Handlungsanweisungen zur Debatte, sondern die Frage, ob ihre Umsetzung unter einem ,schlechten' Herrscher überhaupt möglich sei.

Die Bedeutung der philosophischen Schulen und ihrer Lehrinhalte war im kaiserzeitlichen Rom klar eine andere als im klassischen und hellenistischen Athen. Man könnte von einer Politisierung der philosophischen Kommunikationsräume sprechen. Dies ist eine Tendenz, die sich mit Sicherheit in anderen Städten und in anderen Zeitstellungen ebenfalls beobachten ließe, die sich aber in Rom durch die politische Präsenz der Philosophen bzw. der sie beschreibenden Texte und Autoren besonders deutlich ausgeprägt hat. Cicero

42 Dazu Fuhrer (2000).

43 Mit Thrasea Paetus und Barea Soranus habe Nero, so sagt Tacitus, sogar die „Tugend selbst“ ausrotten wollen (ann. I6,2I,I: virtutem ipsam exscindere concupivit).

44 Vgl. Tacitus' Charakterisierung Thraseas und seines Gesinnungsgenossen und Schwiegersohns Helvidius Priscus in hist. 4,5,2. 
und Tacitus lassen prominente Staatsmänner als Anhänger einer bestimmten secta auftreten oder ein entsprechendes Interesse bekennen und weisen damit den philosophischen Lehren und Schulen eine politische Relevanz zu. Die Kommunikation findet jedoch in einem politisch - mindestens scheinbar - neutralen Raum statt, bei Cicero während der Ferien, außerhalb Roms oder - in einem Fall - an einem Erinnerungsort in Athen, in der Schlussszene von Tacitus' Annalen zwar während der Senatssitzung, aber im Garten in der domus eines Senators. Beide Autoren versetzen damit die an den philosophischen Gesprächen beteiligten nobiles in Kommunikationsräume, die als Alternativen zu denjenigen auf dem Forum markiert sind. Ciceros Szenerien außerhalb Roms eröffnen die Perspektive einer idealisierten Gegenwelt, wie sie vielleicht nie existierte, die sich der politischen Realität der Hauptstadt entzog. Tacitus' philosophisches Hausgespräch führt uns in ein senatorisches Stadthaus, einen philosophischen Kommunikationsraum, der, sobald der Quaestor des Konsuls die Porticus betritt, gestört wird. Mit Thraseas Sterben wird zuletzt auch im cubiculum, dem innersten Bereich des Hauses, wohin er sich mit seinem Schwiegersohn Helvidius Priscus und mit Demetrius zurückgezogen hatte und von wo aus er den Quaestor weiterhin am Geschehen beteiligt, die Kommunikation abgebrochen. 45 Tacitus macht an der Figur des Thrasea deutlich, dass unter der Herrschaft eines moralisch dekadenten Kaisers für die Diskussion und Vermittlung ethischer Werte kein Raum - auch im materiellen Sinn - mehr bleibt.

45 Auch der Text bricht ab; s.o. Anm. 38. - Dafür, dass mit dem in ann. I6,35,I angeredeten iuvenis der Quaestor gemeint sei, den Thrasea zuvor herbeigerufen hatte (propius vocato quaestore), und nicht Helvidius, plädiert Koestermann (I968) 409 . 


\section{Literaturverzeichnis}

Billerbeck (I979)

Margarethe Billerbeck, Der Kyniker Demetrius. Ein Beitrag zur Geschichte der frühkaiserzeitlichen Popularphilosophie, Leiden.

\section{Braund (2009)}

Susanna Braund, Seneca, De clementia, ed. with translation and commentary, Oxford.

\section{Eigler (1996)}

Ulrich Eigler, „Von der Platane im ,Phaidros‘ zur Eiche des Marius - Vergangene Zukunft in Ciceros ,De legibus““, in: Martin Flashar et al. (Hgg.), Retrospektive. Konzepte von Vergangenheit in der griechisch-römischen Antike, München, I37-I46.

\section{Fuhrer (2000)}

Therese Fuhrer, „The Philosopher as Multi-Millionaire: Seneca on Double Standards“, in: Karla Pollmann (Hg.), Double Standards in the Ancient and Medieval World, Göttingen, 2OI-2I9.

\section{Fuhrer (2012)}

Therese Fuhrer, „Filosofia a Roma“, in: Lorenzo Perilli u. Daniela Taormina (Hgg.), La filosofia antica. Itinerario storico e testuale, Turin, 4I7-44I.

\section{Galtier (2002)}

Fabrice Galtier, „L'opposition symbolique des figures de Néron et Thrasea Paetus (Annales XVI,2I-35)“, Latomus 268, 3I2-32I.

\section{Gawlick u. Görler (1994)}

Günter Gawlick u. Woldemar Görler, „Cicero“, in: Hellmut Flashar (Hg.), Grundriss der Geschichte der Philosophie. Die Philosophie der Antike, Bd. 4: Die hellenistische Philosophie, 2. Halbband, Basel, 99I-II68.

\section{Gehrke (1998)}

Hans-Joachim Gehrke, „Theorie und politische Praxis der Philosophen im Hellenismus“, in: Wolfgang Schuller (Hg.), Politische Theorie und Praxis im Altertum, Darmstadt, I0o-I2I.

\section{Gnilka (1979)}

Christian Gnilka, „Ultima verba“, JbAC 22, 5-2I.

\section{Görler (I994)}

Woldemar Görler, „Älterer Pyrrhonismus - Jüngere Akademie - Antiochos aus Askalon“, in: Hellmut Flashar (Hg.), Grundriss der Geschichte der Philosophie. Die Philosophie der Antike, Bd. 4: Die hellenistische Philosophie, 2. Halbband, Basel, 7I7-989.

\section{Griffin (1989)}

Miriam Griffin, „Philosophy, Politics, and Politicians at Rome“, in: Miriam Griffin u. Jonathan Barnes (Hgg.), Philosophia Togata. Essays on Philosophy and Roman Society, Oxford, I-37. 


\section{Haake (2007)}

Matthias Haake, Der Philosoph in der Stadt. Untersuchungen zur öffentlichen Rede über Philosophen und Philosophie in den hellenistischen Poleis, München.

\section{Hadot (2003)}

Ilsetraut Hadot, „Der philosophische Unterrichtsbetrieb in der römischen Kaiserzeit“, RhM I46, 49-7I.

\section{Koestermann (1968)}

Erich Koestermann, Cornelius Tacitus, Annalen, Bd. 4: Buch I4-16, Heidelberg.

\section{Lorenz (1965)}

Thuri Lorenz, Galerien von griechischen Philosophen- und Dichterbildnissen bei den Römern, Mainz.

\section{Maier (1985)}

Barbara Maier, Philosophie und römisches Kaisertum. Studien zu ihren wechselseitigen Beziehungen in der Zeit von Caesar bis Mark Aurel, Diss. Wien.

\section{Malitz (I988)}

Jürgen Malitz, „Philosophie und Politik im frühen Prinzipat“, in: Hans Werner Schmidt u. Peter Wülfing (Hgg.), Antikes Denken - Moderne Schule. Beiträge zu den antiken Grundlagen unseres Denkens, Heidelberg, I5I-I79.

\section{Meggle ( ${ }^{2}$ I997)}

Georg Meggle, Grundbegriffe der Kommunikation, Berlin/New York.

\section{Raaflaub (1987)}

Kurt A. Raaflaub, „Grundzüge, Ziele und Ideen der Opposition gegen die Kaiser im I. Jh. n. Chr.: Versuch einer Standortbestimmung“, Entr. Fond. Hardt 33, I-63.

Radice u. Ramelli (2008)

Roberto Radice u. Ilaria Ramelli, Stoici Romani minori, Milano.

\section{Rawson (1989)}

Elizabeth Rawson, „Roman Rulers and the Philosophic Adviser“, in: Miriam Griffin u. Jonathan Barnes (Hgg.), Philosophia Togata. Essays on Philosophy and Roman Society, Oxford, 233-257.

\section{Roller (200I)}

Matthew B. Roller, Constructing autocracy. Aristocrats and emperors in Julio-Claudian Rome, Princeton.

\section{Shaw (1985)}

Brent Shaw, „The Divine Economy: Stoicism as Ideology“, Latomus 44, I6-54.

\section{Strasburger (1990)}

Hermann Strasburger, Ciceros philosophisches Spätwerk als Aufruf gegen die Herrschaft Caesars, hrsg. von Gisela Strasburger, Hildesheim etc. 
Tandoi (1966)

Vincenzo Tandoi, „Morituri verba Catonis II“, Maia I8, 20-4I.

Trapp (2007/2008)

Michael Trapp, Philosophy in the Roman Empire. Ethics, Politics and Society, Aldershot.

Vielberg (1987)

Meinolf Vielberg, Pflichten, Werte, Ideale. Eine Untersuchung zu den Wertvorstellungen des Tacitus, Stuttgart.

Wassmann (1996)

Herbert Wassmann, Ciceros Widerstand gegen Caesars Tyrannis. Untersuchungen zur politischen Bedeutung der philosophischen Spätschriften, Bonn.

Zadorojnyi (2007)

Alexei V. Zadorojnyi, „Cato's Suicide in Plutarch“, CQ 57, 216-230.

Zetzel (1995)

James E.G. Zetzel, Cicero, De re publica. Selections, Cambridge.

Zoll (1962)

Gallus Zoll, Cicero Platonis Aemulus. Untersuchung über die Form von Ciceros Dialogen, besonders von De oratore, Diss. Zürich. 